\title{
Atomic-scale one-dimensional materials identified from graph theory
}

\section{Shunning Li}

Peking University Shenzhen Graduate School

\section{Zhefeng Chen}

Peking University Shenzhen Graduate School

\section{Zhi Wang}

Peking University Shenzhen Graduate School

\section{Mouyi Weng}

Peking University Shenzhen Graduate School

Jianyuan Li

Peking University Shenzhen Graduate School

\section{Mingzheng Zhang}

Peking University Shenzhen Graduate School

Jing Lu

Peking University

\section{Kang Xu}

United States Army Research Laboratory

Feng Pan ( $\nabla$ panfeng@pkusz.edu.cn )

Peking University Shenzhen Graduate School https://orcid.org/0000-0002-8216-1339

\section{Article}

Keywords: two-dimensional (2D) atomic layers, one-dimensional (1D) atomic chains, zero-dimensional (OD) atomic clusters

Posted Date: February 3rd, 2021

DOl: https://doi.org/10.21203/rs.3.rs-153994/v1

License: (c) (1) This work is licensed under a Creative Commons Attribution 4.0 International License. Read Full License 


\title{
Atomic-scale one-dimensional materials identified from graph theory
}

Shunning $\mathrm{Li}^{1 \dagger}$, Zhefeng Chen ${ }^{1 \dagger}$, Zhi Wang ${ }^{1 \dagger}$, Mouyi Weng ${ }^{1 \dagger}$, Jianyuan $\mathrm{Li}^{1}$, Mingzheng Zhang ${ }^{1}$, Jing Lu ${ }^{2}$, Kang Xu ${ }^{3}$, Feng Pan $^{1 *}$

${ }^{1}$ School of Advanced Materials, Peking University, Shenzhen Graduate School, Shenzhen 518055, China

${ }^{2}$ State Key Laboratory of Mesoscopic Physics and Department of Physics, Peking University, Beijing 100871, China

${ }^{3}$ Electrochemistry Branch, Sensor and Electron Devices Directorate, Power and Energy Division, US Army Research Laboratory, Adelphi, MD, USA

†These authors contributed equally to this work: Shunning Li, Zhefeng Chen, Zhi Wang, Mouyi Weng.

*E-mail: panfeng@pkusz.edu.cn

\begin{abstract}
The past decades have witnessed an exponential growth in the discovery of functional materials, benefited from our unprecedented capabilities in characterizing their structure, chemistry, and morphology with the aid of advanced imaging, spectroscopic and computational techniques. Among these materials, atomic-scale low-dimensional compounds, as represented by the two-dimensional (2D) atomic layers, one-dimensional (1D) atomic chains and zero-dimensional (0D) atomic clusters, have long captivated scientific interest due to their unique topological motifs and exceptional properties. Their tremendous potentials in various applications make it a pressing urgency to establish a complete
\end{abstract}


database of their structural information, especially for the underexplored 1D species. Here we apply graph theory in combination with first-principles high-throughput calculations to identify atomic-scale 1D materials that can be conceptually isolated from their parent bulk crystals. In total, two hundred and fifty $1 \mathrm{D}$ atomic chains are shown to be potentially exfoliable. We demonstrate how the lone electron pairs on cations interact with the $p$-orbitals of anions and hence stabilize their edge sites. Data analysis of the 2D and 1D materials also reveals the dependence of electronic band gap on the cationic percolation network determined by graph theory. The library of 1D compounds systematically identified in this work will pave the way for the predictive discovery of material systems for quantum engineering, and can serve as a source of stimuli for future data-driven design and understanding of functional materials with reduced dimensionality. 
In 1735 , driven by the belief that there should be a standard way of categorization for everything in the natural world, Carolus Linnaeus published Systema Naturae ${ }^{1}$, which was the very first effort to establish scientific classifications of all species from minerals, plants to animals, in order to understand their underneath connections. Confined by his time, this taxonomy was mainly based on superficial observations of visible properties, shapes and traits of the objects, without consideration for the structural and genetic connectivity between these diversified species. Thus, despite its commanding and sustained success in biological kingdoms, the categorization codes are far from being applicable to the field of minerals, because our knowledge about materials and chemistries has been completely reshaped in the last century by the advent of quantum theory and the related explanation of how atoms and molecules are bonded. Up to now, there have been a total of over one hundred thousand inorganic compounds registered in Inorganic Crystal Structure Database ${ }^{2}$ (ICSD) with detailed information of lattice parameters and atomic coordinates. The current categorization of these compounds is mainly based on space groups, which is, however, incapable of evaluating the similarity and relationship among different structures. With the development of mathematical tools, there emerges an effort to encode crystal structures via graph theory $y^{3,4}$, where the constructed structure graphs contain only the topological information of how atoms are connected to each other, while disregarding information about symmetry, atomic distances and bond angles that have a high reliance on the measurement of atomic position. Several pioneering works have shown the advance of structure graphs towards defining the topological features of periodic structures and predicting properties such as formation energy and band gap ${ }^{5-9}$. In this work, we extend the efforts to an unprecedented magnitude by demonstrating that a graph-theory-based categorization of inorganic compounds can enable us to extract the disconnected structural blocks in crystals, and to identify atomic-scale low-dimensional 
materials (LDMs), especially the one-dimensional (1D) atomic chains, via the classification of these structural blocks.

As compared with conventional nanomaterials (nanosheets, nanofibers and quantum dots) that may possess unsaturated dangling bonds, the atomic-scale LDMs are characterized by fully terminated surfaces, which are chemically inert and thus confine the intrinsic properties and functionalities of the $\mathrm{LDMs}^{10,11}$. In recent years, with the aid of state-of-the-art high-throughput first principles calculations, a multitude of atomic-scale LDM candidates have been discovered, predominantly in the form of twodimensional (2D) atomic layers ${ }^{12-19}$. Most of them are either identified via top-down exfoliation of existing bulk materials, or anticipated by bottom-up chemical substitutions of already-synthesized 2D compounds ${ }^{16-18}$. The newly established $2 \mathrm{D}$ materials databases have ignited research interest in the virtual screening for their potential applications as catalysts ${ }^{20-22}$, ferromagnets ${ }^{23}$, field-effect transistors and other electronic devices ${ }^{24,25}$, which dramatically expands the possibilities of nanoscale design in a variety of research frontiers. In contrast, other LDMs, i.e. 1D atomic chains ${ }^{26-29}$ and zero-dimensional (0D) atomic clusters ${ }^{30-32}$, have not received the comparable attention and enthusiasm as 2D materials did from the academic community, for which the lack of a predictive knowledge of $1 \mathrm{D}$ and $0 \mathrm{D}$ structures has been mainly responsible. It is worth mentioning that $1 \mathrm{D} \mathrm{Si} / \mathrm{Ge} / \mathrm{III}-\mathrm{V}$ nanowires ${ }^{33-35}$ are widely regarded as one of the most competitive channel materials of the next-generation transistors to supplant the Fin channels. Presumably, the dangling-bond-free semiconducting 1D atomic chains, if available, would outperform these nanowires in a gate-all-around device. Under this context, it would be of critical importance to establish a correlation between intrinsic properties and salient topological features of the atomic-scale 1D materials via graph theory.

Herein, a structural analysis based on graph theory and density functional theory (DFT) 
calculations is conducted with the aim of a comprehensive and systematic screening for atomic-scale 2D, 1D and 0D materials. 1D materials are of special interest because their distinct difference from 2D counterparts may have broad appeal to researchers in the area of electronic devices. By categorizing the structural blocks in bulk compounds retrieved from ICSD, 365 1D compounds are identified and over half of them are predicted to be potentially exfoliable from parent bulk crystals. We present specific cases to illustrate the structural inheritance of 1D structures from 2D compounds, and highlight the role of lone electron pair (LEP) on self-passivation at edge sites. Graph theory is further employed to group $2 \mathrm{D}$ and $1 \mathrm{D}$ compounds with similar cation-connection motifs, from which we identify a statistical correlation between the atomic networks and their electronic band structures. The findings in this work sheds light on a new paradigm where the structure-property relationships in materials are captured by the insight into the structure units and their specific connection patterns.

\section{Workflow}

In structure graphs, atoms are represented as nodes and the direct chemical interactions are abstracted as connections between these nodes. The graph-theory-based classification of materials into different geometric categories is a rigorous and unbiased approach for investigating structural prototypes, even if symmetry is broken and fluctuation in bond lengths is huge. To discover atomicscale LDMs from bulk compounds, we first acquire the structure graphs of the available compounds in ICSD (Fig. 1). By discarding error items as well as materials with fractional occupancy at lattice sites, there remain a total of 88159 unique compounds. For each compound, neighboring atoms are searched relying on the covalent, ionic or metallic radii of the corresponding atoms. The choice of which kind of radius to adopt is in accord with the electronegativity of both elements. The atomic 
adjacency matrix is then constructed, which presents a portrait of the compound and serves as the representative of structure graph for the identification of exfoliable materials (detailed methods are given in Supplementary Information section 1). We use this adjacency matrix to determine the dimensionality of the chemically connected blocks in the bulk material. The $2 \mathrm{D}, 1 \mathrm{D}$ and $0 \mathrm{D}$ structural blocks are successfully identified and isolated to produce a library of LDMs, after which we apply graph theory again to group them into different structural prototypes. The above procedures yield a total of 1194, 365 and 233 unique 2D, 1D and 0D compounds (Supplementary Table 9-11), respectively, and the numbers of the corresponding structural prototypes are 698, 223 and 179. Finally, highthroughput DFT calculations (Supplementary Information section 2) are performed on all the derived LDMs. It should be emphasized that in this work, the chemical bonding is determined robustly by a prior classification of covalent, ionic and metallic interactions between possible neighboring atoms. The proper choice of atomic radii in our identification protocol can enable accurate evaluation of the atomic connectivity and will therefore offer ample opportunities in discovering new LDMs. 


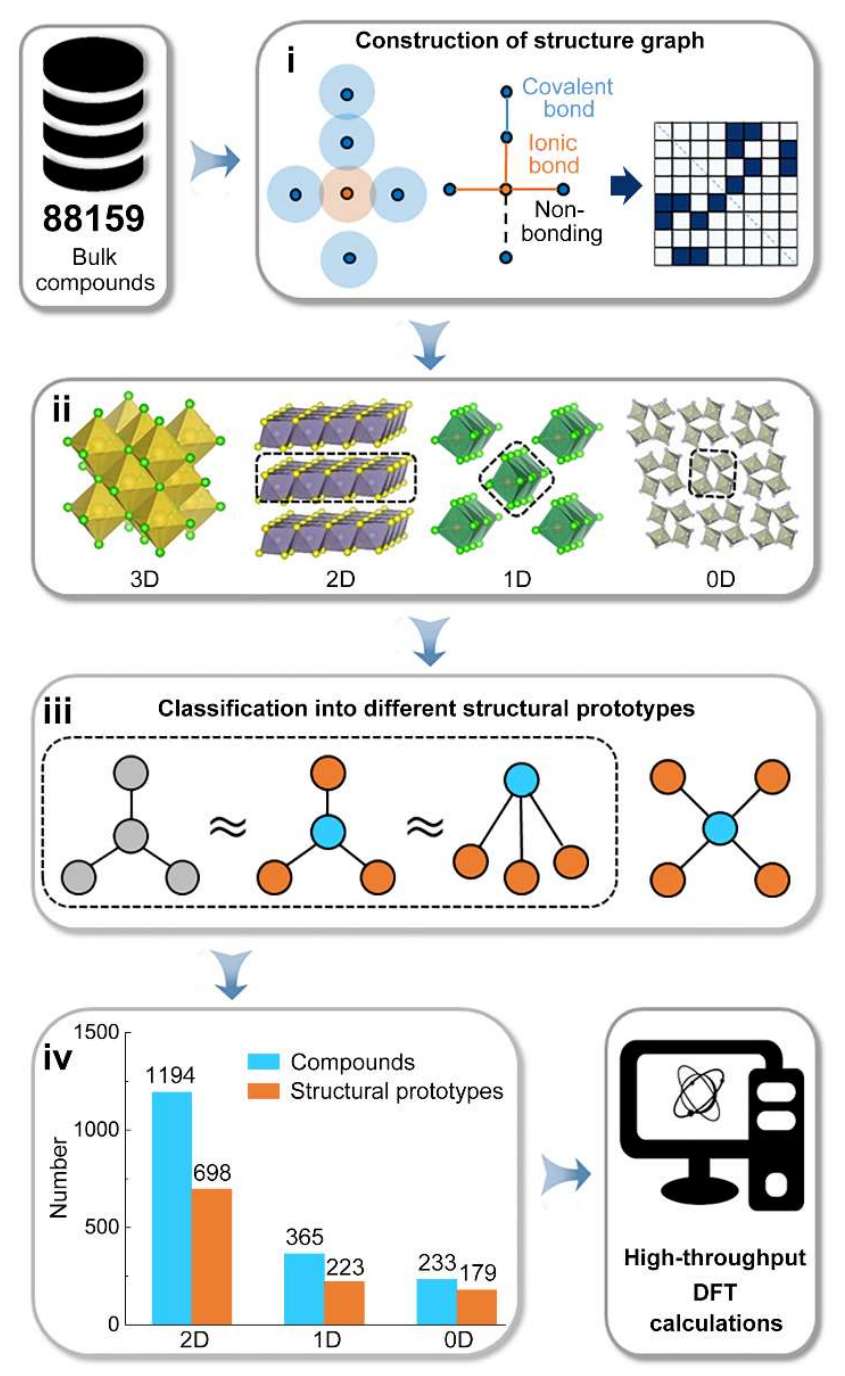

Fig. 1 | An overview of the workflow for LDM identification and classification. Four main steps are included: i, construction of structure graphs for bulk compounds; ii, categorization of the chemically connected structural blocks; iii, classification of the isolated structural blocks with different dimensionality; iv, statistics on the LDMs.

\section{Datasets of 1D and 0D materials}

The exfoliation energy $E_{\text {exf }}$, defined as the energy difference between the LDMs and their parent bulk compounds ${ }^{36,37}$, is computed to evaluate the feasibility of exfoliation. Figure $2 \mathrm{a}-\mathrm{c}$ presents the calculated $E_{\text {exf }}$ as a function of the interatomic distance $\left(d_{\text {block }}\right)$ between the structural blocks in bulk materials. A considerable number of $2 \mathrm{D}$ and $1 \mathrm{D}$ materials require relatively low energy cost to be 
exfoliated, whereas the exfoliation of the $0 \mathrm{D}$ atomic clusters tends to incur a large energy penalty. Remarkably, we have uncovered 250 1D and 22 0D materials that are as exfoliable as typical 2D materials (threshold of $E_{\text {exf }}$ is chosen from GaS, see Supplementary Table 2). Confidence is therefore lent to the systematic analysis of the largely unexplored realm of $1 \mathrm{D}$ atomic chains and $0 \mathrm{D}$ atomic clusters. It is also worth noting that a small negative relationship between $E_{\text {exf }}$ and $d_{\text {block }}$ arises in 1D and $0 \mathrm{D}$ compounds, in contrast to the apparently weak relationship displayed in 2D counterparts. This suggests that with lower dimensionalities, a sufficient distance between structural blocks is more strongly required to counterbalance the destabilization caused by the large surface exposure.

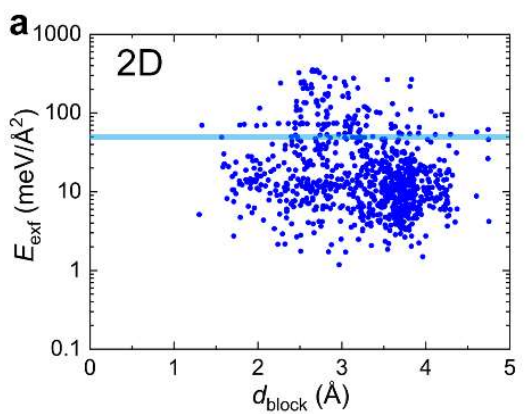

d

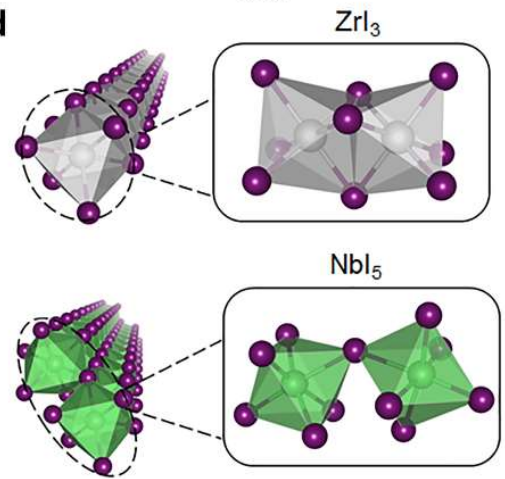

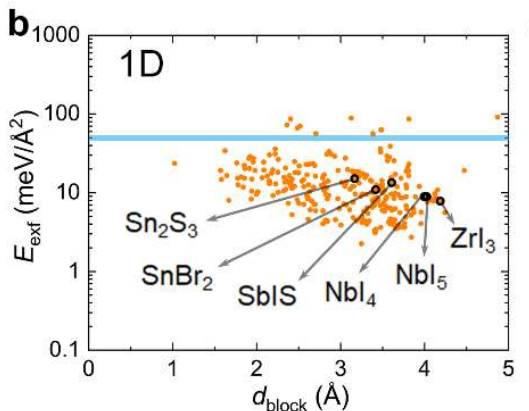

$\mathrm{Nbl}_{4}$

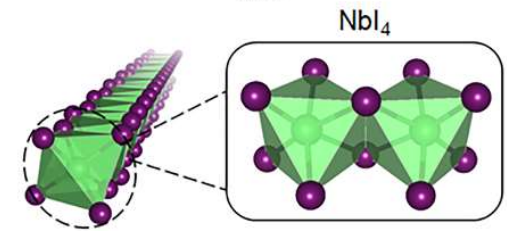

SblS

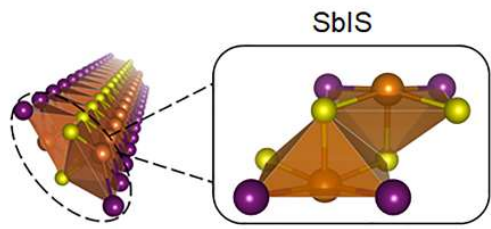

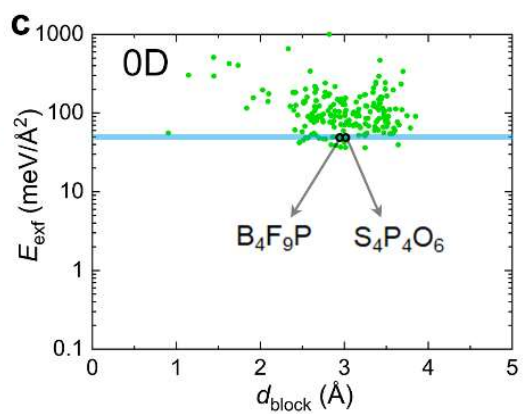

e
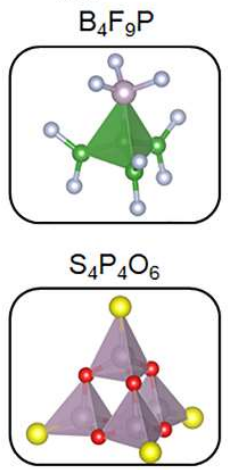

Fig. 2 | Exfoliation energy of LDMs and structure of representative 1D/0D materials. a-c, Exfoliation energy per surface area for 2D (a), 1D (b) and 0D (c) compounds. The blue line indicates a threshold defined by the highest value of $E_{\text {exf }}$ for the experimentally accessible 2D compounds. d, e, Representative structural prototypes of 1D atomic chains (d) and 0D atomic clusters (e).

Many of the 1D compounds lie in a few easily recognizable structural motifs. Figure $2 \mathrm{~d}$ shows four 
representatives of them, featured by face-sharing, edge-sharing and vertex-sharing $\mathrm{MX}_{6}$ octahedra or MX 5 half-octahedra (M: cation; X: anion). In Supplementary Information section 3, we present examples of other structural prototypes, each of which comprises regular or semiregular coordination polyhedra (CP). For those with regular $\mathrm{CP}$, the absence of dangling bonds on the cations and the closeshell electronic structure of the anions can endow their parent bulk materials with a highly anisotropic character, whereby the physical and chemical properties can remain unaffected by grain boundaries and surfaces parallel to the 1D chain. As a result, it is desirable to fabricate electronic devices with 1D compounds with regular $\mathrm{CP}$ and suitable band gap, which can significantly suppress the scattering from surface roughness and trap states ${ }^{38,39}$, and thus are more advantageous than the $1 \mathrm{D} \mathrm{Si} / \mathrm{Ge} / \mathrm{III}-\mathrm{V}$ nanomaterials in terms of carrier mobility and electrostatic performance. Moreover, we note that although the amount of 1D materials is limited, a significant fraction of them are binary or ternary compounds with simple structures. This could allow for combinatorial substitution by other elements to augment the diversity of $1 \mathrm{D}$ materials.

Two 0D compounds characteristic of covalent bonding are also exemplified in Fig. 2e. Versatile structures of 0D compounds are demonstrated in Supplementary Table 5. It is found that isostructural $0 \mathrm{D}$ compounds are much rarer than the $2 \mathrm{D}$ and $1 \mathrm{D}$ counterparts, which is attributable to the higher flexibility in the number of constituent atoms in a cluster, as imposed by the removal of periodic boundary conditions in all directions. The repository of 0D materials shows a high prevalence for covalent bonding between main-group elements, including boron, carbon and phosphorus (Supplementary Table 6-8), with numerous architectures existing in various compositions. The assembly of these atomic clusters into their parent bulk materials can present fascinating examples for the study of the packing of fullerene-like molecules ${ }^{30,31}$. 


\section{Self-passivation in atomic chains}

The semiregular $\mathrm{CP}$ in some of the 1D atomic chains have led us to explore the self-passivation mechanism of their unsaturated dangling bonds, which could open the possibility of manipulating the dimensionality of materials. To this end, we screen the complete collection of 2D and 1D compounds for cases where the 2D and 1D structures are well correlated. This criterion is met by the $2 \mathrm{D} \mathrm{SnS}_{2}$ and the $1 \mathrm{D} \mathrm{Sn}_{2} \mathrm{~S}_{3}$, as well as $1 \mathrm{D} \mathrm{SnBr}_{2}$ (Fig. 3a-c). Both atomic chains contain bivalent Sn ions. As compared to the $\mathrm{SnS}_{6}$ octahedron in $\mathrm{SnS}_{2}$, the edge of $\mathrm{Sn}_{2} \mathrm{~S}_{3}$ is featured by trigonally coordinated $\mathrm{Sn}^{2+}$ (valency is deduced from Bader charge in Supplementary Table 3), while in $\mathrm{SnBr}_{2}, \mathrm{Sn}^{2+}$ ion sits at a pyramidal site. Apparently, each of the atomic chains adopts a structure that can be regarded as truncated from the 1T-phase $\mathrm{SnS}_{2}$, which leaves unsaturated dangling bonds free to interact with external atoms. This seems to be at odds with the calculated $E_{\text {exf }}$ that indicates the feasibility of isolating $\mathrm{Sn}_{2} \mathrm{~S}_{3}$ and $\mathrm{SnBr}_{2}$ atomic chains from bulk phases (Fig. 2b). To clarify the electronic origin of this self-passivation, we calculated the density of states (DOS) localized on different atomic sites, as shown in the insets of Fig. 3b,c. The Sn $5 s$ orbitals occupy deep energies far from the valence band maximum ( $\sim 6 \mathrm{eV}$ apart), indicating that the LEP of $\mathrm{Sn}^{2+}$ ion is relatively non-labile. To trace the location of LEP, we calculated the electron localization function (ELF) ${ }^{40}$ for $\mathrm{Sn}_{2} \mathrm{~S}_{3}$ and $\mathrm{SnBr}_{2}$ (Fig. 3df), with $\mathrm{SnS}_{2}$ shown for comparison. A local maximum of the ELF is observed at the edges of the atomic chains, pinpointing the lobelike LEP that deviates from the central atom nucleus of $\mathrm{Sn}^{2+}$. We note that the LEP is stereochemically active and therefore participates in the coordination polyhedron of $\mathrm{Sn}^{2+}$, resembling the cases of $\mathrm{Pb}^{2+}$ and $\mathrm{Bi}^{3+}$ in lone pair containing compounds ${ }^{41}$. 
a $\mathrm{SnS}_{2}$
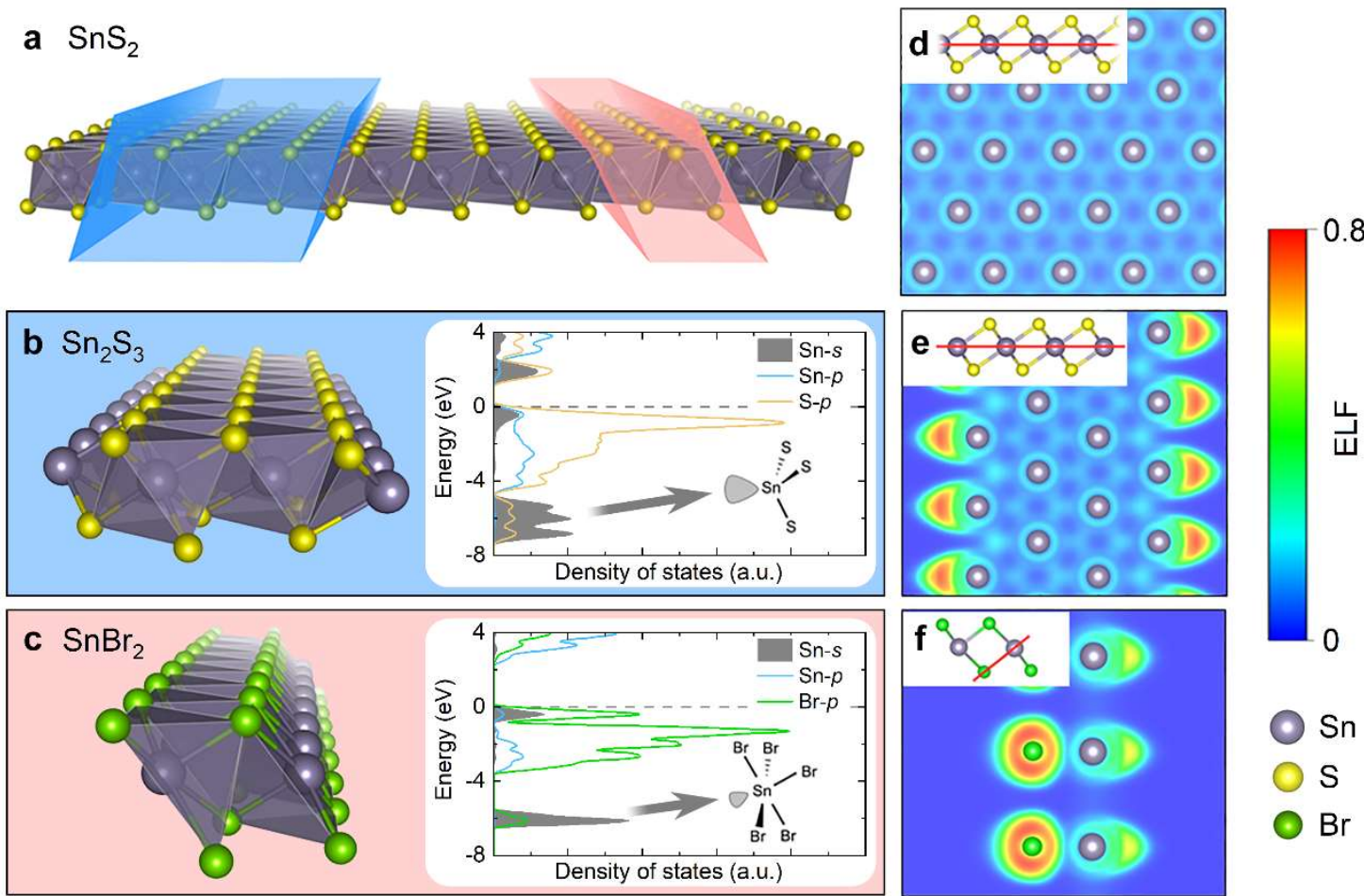

Fig. 3 | Self-passivation via LEP on $\mathrm{Sn}^{2+}$. a, Structure of $\mathrm{SnS}_{2}$ monolayer. The blue and red frames denote the structural inheritance of $\mathrm{Sn}_{2} \mathrm{~S}_{3}$ and $\mathrm{SnBr}_{2}$ from $\mathrm{SnS}_{2}$, respectively. b, c, Structures of $\mathrm{Sn}_{2} \mathrm{~S}_{3}$ (b) and $\mathrm{SnBr}_{2}$ (c) atomic chains and their electronic DOS. Fermi level is set to zero. States in the energy range of $-7 \sim-5 \mathrm{eV}$ correspond to the $5 s \mathrm{LEP}$ on $\mathrm{Sn}^{2+}$ ions. d-f, ELFs of $\mathrm{SnS}_{2}(\mathbf{d}), \mathrm{Sn}_{2} \mathrm{~S}_{3}(\mathbf{e})$ and $\mathrm{SnBr}_{2}$ (f) in the planes designated by the red lines in the insets. Local maximum of ELF around Sn atom indicates the LEP.

Due to the spherically symmetric nature of $s$ orbitals, there must be hybridization between $\mathrm{Sn} 5 s$ and other states with $p$ character in $\mathrm{Sn}_{2} \mathrm{~S}_{3}$ and $\mathrm{SnBr}_{2}$, otherwise the LEP will not form lobe shape ${ }^{42}$. As can be inferred from the significant overlap between Sn-s and S/Br- $p$ orbitals in the DOS (Fig. 3b,c), the Sn $5 s$ and anion $p$ states are energetically degenerate in these atomic chains. The crystal orbit Hamiltonian population shown in Supplementary Fig. 10 reveals a bonding-antibonding fashion between $\mathrm{Sn}$ and $\mathrm{S} / \mathrm{Br}$ in the energy range of $-7 \sim-5 \mathrm{eV}$ (bonding) and just below the Fermi level (antibonding), suggesting that the majority of $\mathrm{Sn} 5 s$ states is involved in bond formation with $\mathrm{S} / \mathrm{Br}-p$ 
states. The charge density (Supplementary Fig. 11) between -7 and $-5 \mathrm{eV}$ also shows a considerable proportion of electrons distributing between $\mathrm{Sn}$ and $\mathrm{S} / \mathrm{Br}$. These results demonstrate that in addition to fulfilling the role of a ligand for the undercoordinated $\mathrm{Sn}^{2+}$ ion, the LEP in $\mathrm{Sn}_{2} \mathrm{~S}_{3}$ and $\mathrm{SnBr}_{2}$ can endow high stability to the edges of both materials via strong coupling with the anion $p$ states. It is therefore expected that edge doping of main-group elements with LEP, such as $\mathrm{Sn}^{2+}, \mathrm{Pb}^{2+}$ and $\mathrm{As}^{3+}$, will stabilize the $2 \mathrm{D}$ nanoribbons and can therefore serve as a powerful strategy towards harnessing the dimensionality and chemical properties of LDMs.

\section{Correlation between cation connectivity and electronic band gap}

Graph theory can also be introduced to unveil the structure-property relationships of LDMs. Here, we examine how the dimensionality and the cation connectivity exert an influence on the electronic band structure of the 2D and 1D materials. To better describe the structures, we first summarize the numbers of face-sharing, edge-sharing and vertex-sharing linkages between cation-centered CP, as detailed in Supplementary Information section 4. Edge-sharing linkage predominates over other connectivity patterns, regardless of the dimensionality of the LDMs. Intimate connection between cations is defined as either direct connection via metallic bonds or face/edge-sharing linkages of the corresponding polyhedra. On the contrary, vertex-sharing linkage underlies the disconnection between adjacent cations. We discover many $2 \mathrm{D}$ and $1 \mathrm{D}$ materials in which the interconnected cations form a percolation network (for 1D materials it will be a percolation chain). Examples include $\mathrm{MoS}_{2}, \mathrm{Bi}_{2} \mathrm{Te}_{3}$, $\mathrm{W}_{2} \mathrm{~N}_{3}$ atomic layers (Supplementary Fig. 13), and $\mathrm{ZrI}_{3}, \mathrm{NbI}_{4}$ and $\mathrm{SbIS}$ atomic chains presented in Fig. $2 \mathrm{~d}$. This percolation property can serve as a powerful classification rule to distinguish between densely and loosely packed sublattices of cations (Fig. 4a,b). Notably, over half of the 2D and 1D compounds 
are found to possess a cationic percolation network. Although the ratio of vertex-sharing linkage is nearly the same at both dimensionalities, 2D materials have a higher fraction of such network than 1D atomic chains (Supplementary Fig. 12,15). This result implies that the cation connectivity can be biased by the dimensionality of compounds. We would like to stress that the above classification scheme is not guided by interatomic distances but by the structure graphs of the LDMs.
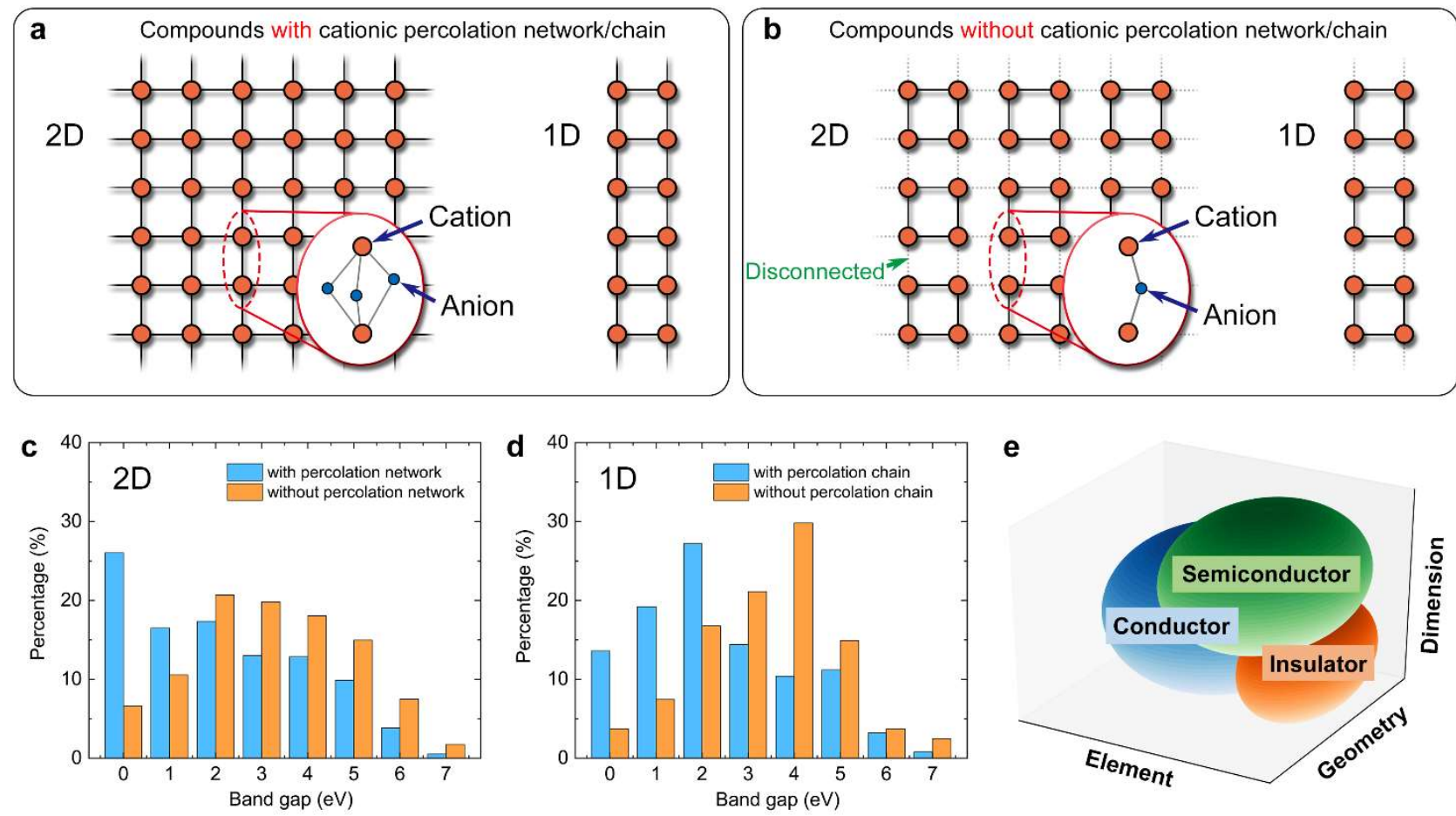

Fig. 4 | Influence of cation connectivity on electronic band gap. a, b, Schematic illustration of 2D and 1D structures with (a) or without (b) a cationic percolation network/chain. $\mathbf{c}, \mathbf{d}$, The distribution of band gap for 2D (c) and 1D (d) compounds, each divided into two groups by the existence of a cationic percolation network. e, Modulation of electronic structure by tuning constituent element, geometry and dimensionality of the material.

Figure $4 \mathrm{c}, \mathrm{d}$ presents the distribution of band gap for 2D and 1D compounds with and without a cationic percolation network. As apparent from the histograms, the group featuring cationic percolation 
network tends to yield a smaller band gap than its counterpart. The existence of a cationic percolation network would correspond to a higher packing density of the cations, and thereby a closer proximity between cation-centered $\mathrm{CP}$, which can be regarded as the structure units in a compound ${ }^{43}$. In this scenario, we can expect there to be a higher degree of overlap in the atomic orbitals between adjacent structure units, whereby the bandwidth would be widened and the band gap narrowed down. For 1D atomic chains, the band gaps are generally distributed at higher values than the $2 \mathrm{D}$ compounds, thus confirming that the reduction of dimensionality can statistically affect the electronic structure by an enhancement of quantum confinement effects. Although many studies on atomic-scale LDMs have shown the dependence of their electronic properties on the constituent elements and structure units ${ }^{20-}$ ${ }^{25}$, none shows the essential role played by the dimensionality and cation connectivity. The above results demonstrate that a combination of elemental, geometrical and dimensional modulation can be incorporated to mediate the electronic structure of LDMs (Fig. 4e), and that the exploration of cationic percolation network would represent a viable avenue for the design of low-dimensional conductors, semiconductors and insulators for use in the fabrication of electronic devices.

\section{Conclusions}

This work presents an important step towards the comprehensive and systematic screening and classification of atomic-scale low-dimensional compounds - atomic layers, chains and clusters with the application of graph theory that permits the encoding of topological information for all structures. The algorithmic approach is applied to the experimentally known compounds from ICSD, yielding a variety of structural blocks with different dimensionality. Many of them are expected to be exfoliable from bulk phases, and their isolation into LDMs could introduce a promising platform to 
expedite the experimental investigations in quantum confinement effects. The full collection of 1D atomic chains displays a fascinating structural and compositional diversity. Enlightened from the examples of 1D compounds whose structures contain semiregular $\mathrm{CP}$ and bear a strong resemblance to certain prototypes of 2D compounds, we propose a novel strategy based on LEPs of $s$ orbitals to passivate the dangling bonds, thus offering opportunities for the control of dimensionality. Given the low-lying energy levels of these LEPs, our strategy can overcome the shortcomings of conventional nanoribbons and nanorods such as poor stability and the formation of unfavorable edge states. We also employ the structure graphs to distinguish between 2D/1D compounds with and without a cationic percolation network. It is revealed that the emergence of such a network would generally be accompanied by a relatively narrow electronic band gap, implicating the essential role of cation connectivity in electronic-structure predictions. Putting these insights together suggests that the structure-property relationships in atomic-scale 1D materials are to a large extent dependent on their topological features, which, as portrayed in the structure graphs, provide a new perspective on the characterization of structures with reduced dimensionality.

\section{Acknowledgements}

This work was financially supported by National Key R\&D Program of China (2016YFB0700600), and Shenzhen Science and Technology Research Grant (No. JCYJ20200109140416788).

\section{Author contributions}

F.P. conceived and supervised this project. S.L., Z.C., Z.W. and M.W. performed the calculations. S.L., Z.C., Z.W., Jianyuan Li, M.Z. and F.P. analyzed the calculated results and built the database for LDMs. 
Z.W. and M.W. wrote the code for constructing the structure graphs. Jing Lu assisted with the electronic structure analysis. S.L., K.X. and F.P. co-wrote the main text. All authors discussed the results and commented on the manuscript.

Competing interests The authors declare no competing interests.

\section{Additional information}

Supplementary information is available for this paper.

Correspondence and requests for materials should be addressed to F.P.

\section{References}

1 Linnaeus, C. Systema Naturae: sive Regna Tria Naturae Systematice Proposita per Classes, Ordines, Genera, \& Species (Haak, 1735).

2 Inorganic Crystal Structure Database (ICSD); http://www.fiz-karlsruhe.com/icsd.html

3 Bonchev, D. \& Rouvray, D. H. Chemical Graph Theory: Introduction and Fundamentals (Abacus Press, 1991).

4 King, R. B. Applications of Graph Theory and Topology in Inorganic Cluster and Coordination Chemistry (CRC Press, 1992).

5 Isayev, O. et al. Universal fragment descriptors for predicting properties of inorganic crystals. Nat. Commun. 8, 15679 (2017).

6 Xie, T. \& Grossman, J. C. Crystal graph convolutional neural networks for an accurate and interpretable prediction of material properties. Phys. Rev. Lett. 120, 145301 (2018).

$7 \quad$ Weng, M. et al. Identify crystal structures by a new paradigm based on graph theory for building materials big data. Sci. China-Chem. 62, 982-986 (2019).

8 Chen, C., Ye, W., Zuo, Y., Zheng, C. \& Ong, S. P. Graph networks as a universal machine learning framework for molecules and crystals. Chem. Mater. 31, 3564-3572 (2019).

9 Lu, S. et al. Coupling a crystal graph multilayer descriptor to active learning for rapid discovery of 2D ferromagnetic semiconductors/half-metals/metals. Adv. Mater. 32, 2002658 (2020).

10 Franklin, A. D. Nanomaterials in transistors: From high-performance to thin-film applications. Science 349, aab2750 (2015).

11 Iannaccone, G., Bonaccorso, F., Colombo, L. \& Fiori, G. Quantum engineering of transistors based on 2D materials heterostructures. Nat. Nanotechnol. 13, 183-191 (2018).

12 Novoselov, K. S., Mishchenko, A., Carvalho, A. \& Castro Neto, A. H. 2D materials and van 
der Waals heterostructures. Science 353, aac9439 (2016).

Akinwande, D. et al. Graphene and two-dimensional materials for silicon technology. Nature 573, 507-518 (2019).

Ashton, M., Paul, J., Sinnott, S. B. \& Hennig, R. G. Topology-scaling identification of layered solids and stable exfoliated 2D materials. Phys. Rev. Lett. 118, 106101 (2017).

5 Cheon, G. et al. Data mining for new two- and one-dimensional weakly bonded solids and lattice-commensurate heterostructures. Nano Lett. 17, 1915-1923 (2017).

6 Haastrup, S. et al. The computational 2D materials database: high-throughput modeling and discovery of atomically thin crystals. 2D Mater. 5, 042002 (2018).

1 Mounet, N. et al. Two-dimensional materials from high-throughput computational exfoliation of experimentally known compounds. Nat. Nanotechnol. 13, 246-252 (2018).

Zhou, J. et al. 2DMatPedia, an open computational database of two-dimensional materials from top-down and bottom-up approaches. Sci. Data 6, 86 (2019).

9 Sorkun, M. C., Astruc, S., Koelman, J. M. V. A. \& Er, S. An artificial intelligence-aided virtual screening recipe for two-dimensional materials discovery. npj Comput. Mater. 6, 106 (2020).

Jain, A., Wang, Z. \& Nørskov, J. K. Stable two-dimensional materials for oxygen reduction and oxygen evolution reactions. ACS Energy Lett. 4, 1410-1411 (2019).

1 Karmodak, N. \& Andreussi, O. Catalytic activity and stability of two-dimensional materials for the hydrogen evolution reaction. ACS Energy Lett. 5, 885-891 (2020).

2 Torrisi, S. B., Singh, A. K., Montoya, J. H., Biswas, T. \& Persson, K. A. Two-dimensional forms of robust CO2 reduction photocatalysts. npj 2D Mater. Appl. 4 (2020).

3 Torelli, D., Moustafa, H., Jacobsen, K. W. \& Olsen, T. High-throughput computational screening for two-dimensional magnetic materials based on experimental databases of threedimensional compounds. npj Comput. Mater. 6 (2020).

4 Guo, Y. et al. Eighteen functional monolayer metal oxides: wide bandgap semiconductors with superior oxidation resistance and ultrahigh carrier mobility. Nanoscale Horizons 4, 592-600 (2019).

5 Tian, T. et al. Electronic Polarizability as the Fundamental Variable in the Dielectric Properties of Two-Dimensional Materials. Nano Lett. 20, 841-851 (2020).

6 Kibsgaard, J. et al. Atomic-Scale Structure of Mo6S6 Nanowires. Nano Lett. 8, 3928-3931 (2008).

2 Liu, X. et al. Top-down fabrication of sub-nanometre semiconducting nanoribbons derived from molybdenum disulfide sheets. Nat. Commun. 4, 1776 (2013).

Lin, J. H. et al. Flexible metallic nanowires with self-adaptive contacts to semiconducting transition-metal dichalcogenide monolayers. Nat. Nanotechnol. 9, 436-442 (2014).

9 Liu, X. et al. Direct fabrication of functional ultrathin single-crystal nanowires from quasi-onedimensional van der Waals crystals. Nano Lett. 16, 6188-6195 (2016).

Kong, X.-J., Long, L.-S., Zheng, Z., Huang, R.-B. \& Zheng, L.-S. Keeping the ball rolling: fullerene-like molecular clusters. Accounts Chem. Res. 43, 201-209 (2010).

1 Zhai, H. J. et al. Observation of an all-boron fullerene. Nat. Chem. 6, 727-731 (2014).

2 Geng, L. et al. $\mathrm{Co}_{13} \mathrm{O}_{8}$ - metalloxocubes: a new class of perovskite-like neutral clusters with cubic aromaticity. Natl. Sci. Rev., nwaa201 (2020).

3 Cui, Y., Zhong, Z., Wang, D., Wang, W. U. \& Lieber, C. M. High performance silicon nanowire field effect transistors. Nano Lett. 3, 149-152 (2003). 
34 Xiang, J. et al. Ge/Si nanowire heterostructures as high-performance field-effect transistors. Nature 441, 489-493 (2006).

35 Tomioka, K., Yoshimura, M. \& Fukui, T. A III-V nanowire channel on silicon for highperformance vertical transistors. Nature 488, 189-192 (2012).

36 Bjorkman, T., Gulans, A., Krasheninnikov, A. V. \& Nieminen, R. M. van der Waals bonding in layered compounds from advanced density-functional first-principles calculations. Phys. Rev. Lett. 108, 235502 (2012).

37 Jung, J. H., Park, C. H. \& Ihm, J. A rigorous method of calculating exfoliation energies from first principles. Nano Lett. 18, 2759-2765 (2018).

$38 \mathrm{Ni}$, Z. et al. Performance upper limit of sub-10 nm monolayer $\mathrm{MoS}_{2}$ transistors. Adv. Electron. Mater. 2, 1600191 (2016).

39 Liu, Y., Duan, X., Huang, Y. \& Duan, X. Two-dimensional transistors beyond graphene and TMDCs. Chem. Soc. Rev. 47, 6388-6409 (2018).

40 Silvi, B. \& Savin, A. Classification of chemical bonds based on topological analysis of electron localization functions. Nature 371, 683-686 (1994).

41 Walsh, A., Payne, D. J., Egdell, R. G. \& Watson, G. W. Stereochemistry of post-transition metal oxides: revision of the classical lone pair model. Chem. Soc. Rev. 40, 4455-4463 (2011).

42 Watson, G. W., Parker, S. C. \& Kresse, G. Ab initio calculation of the origin of the distortion of $\alpha$-PbO. Phys. Rev. B 59, 8481-8486 (1999).

43 Zheng, J., Ye, Y. \& Pan, F. 'Structure units' as material genes in cathode materials for lithiumion batteries. Natl. Sci. Rev. 7, 242-245 (2019). 


\section{Figures}
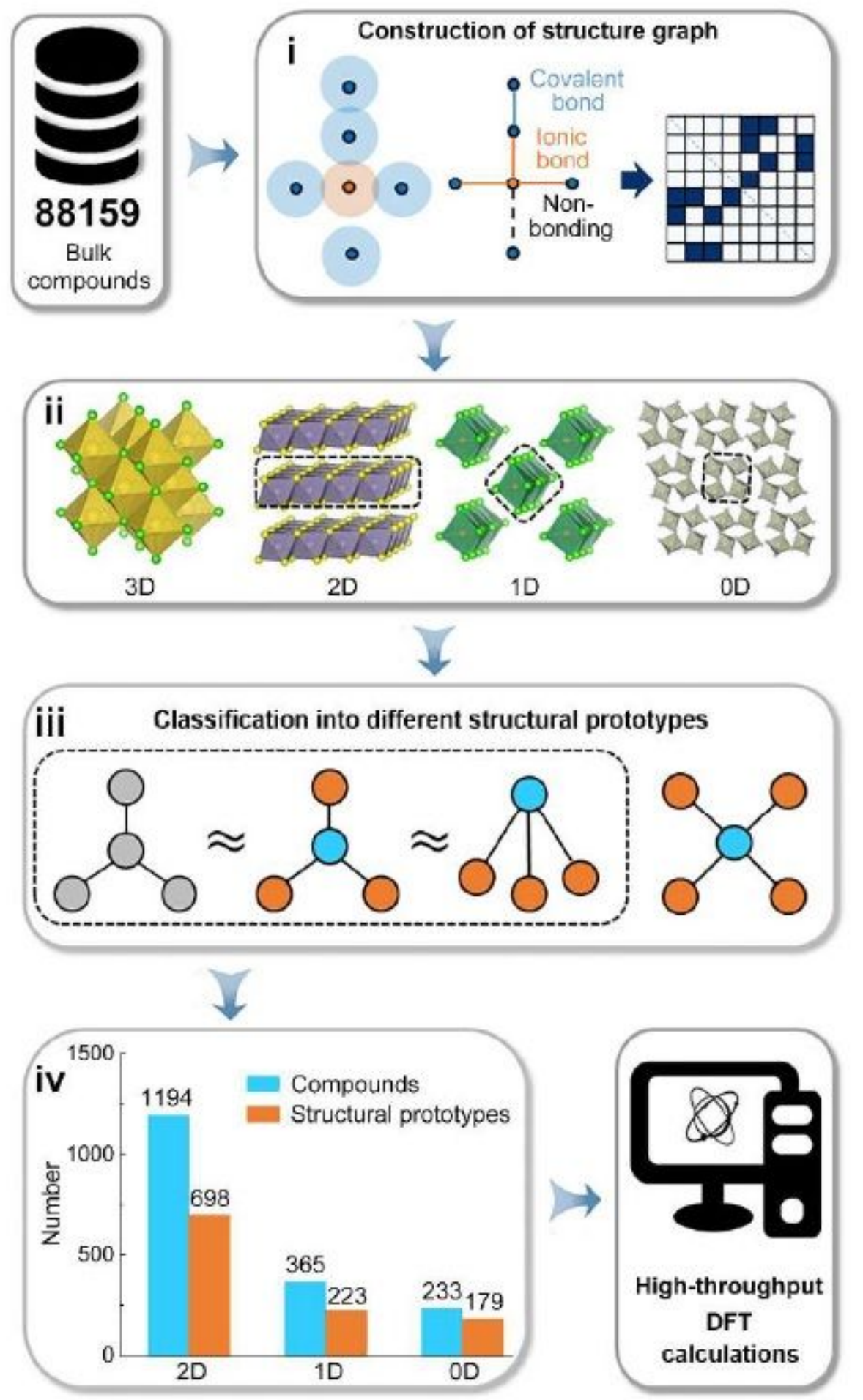

Figure 1

An overview of the workflow for LDM identification and classification. Four main steps are included: i, construction of structure graphs for bulk compounds; ii, categorization of the chemically connected 
structural blocks; iii, classification of the isolated structural blocks with different dimensionality; iv, statistics on the LDMs.
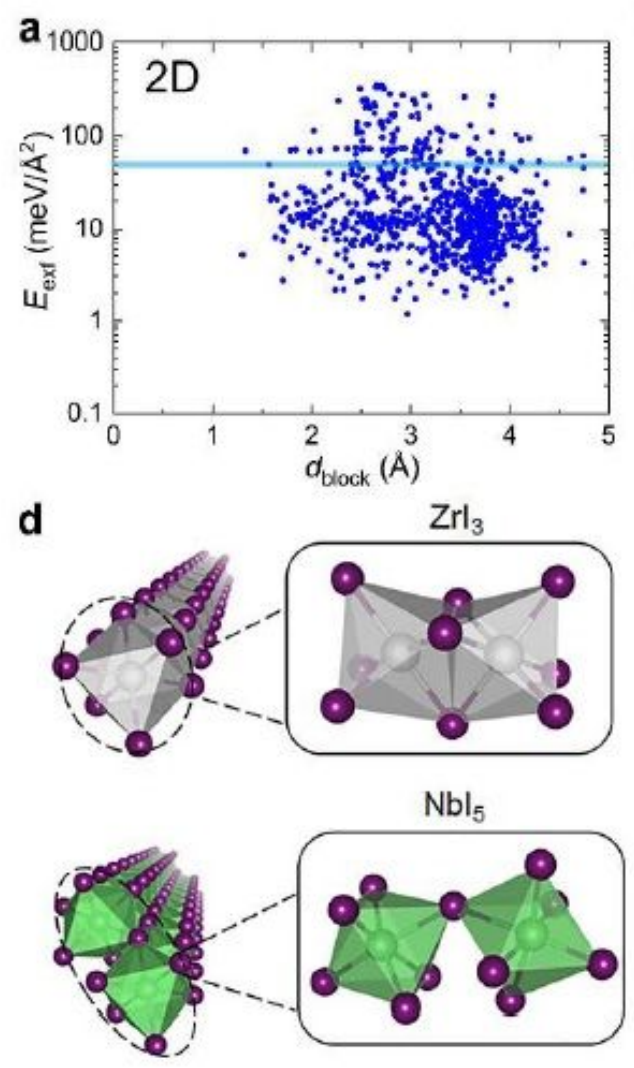
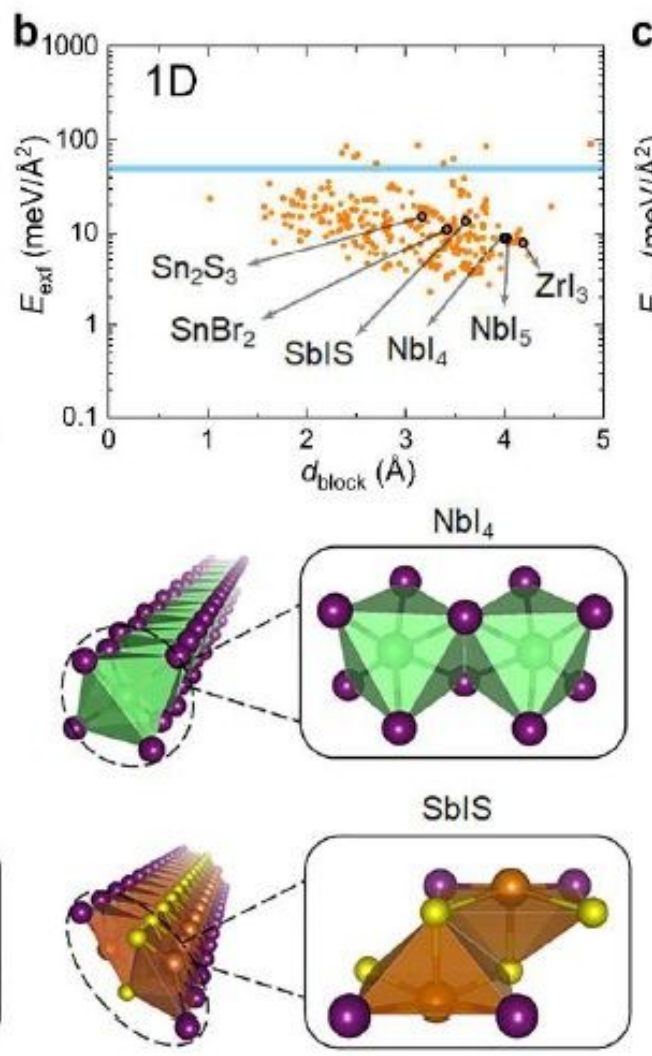

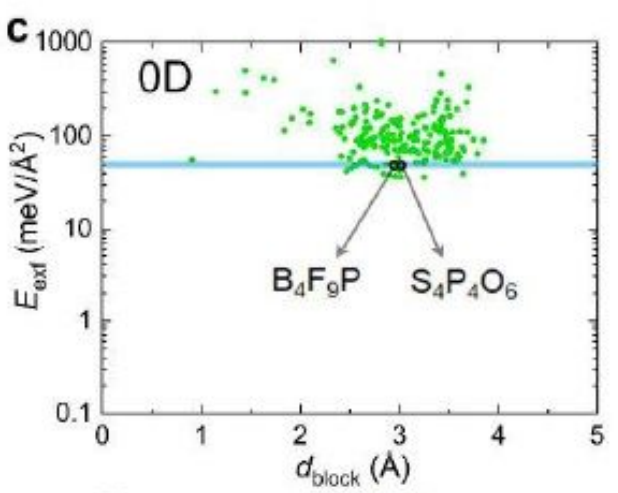

e
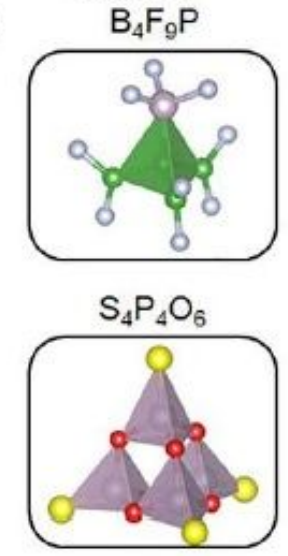

\section{Figure 2}

Exfoliation energy of LDMs and structure of representative 1D/0D materials. a-c, Exfoliation energy per surface area for 2D (a), 1D (b) and OD (c) compounds. The blue line indicates a threshold defined by the highest value of Eexf for the experimentally accessible 2D compounds. $d, e$, Representative structural prototypes of 1D atomic chains (d) and OD atomic clusters (e). 


\section{a $\mathrm{SnS}_{2}$}
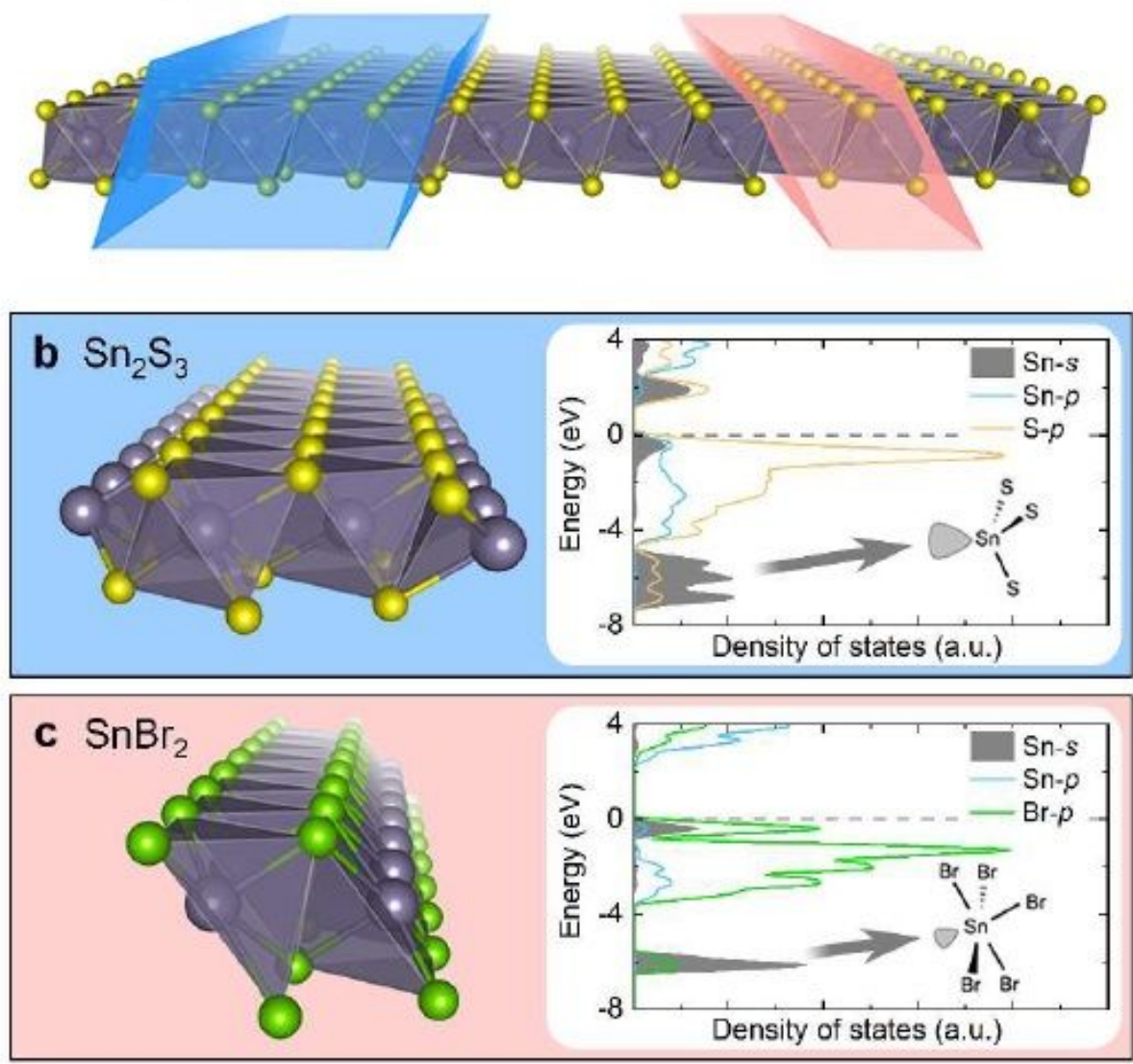
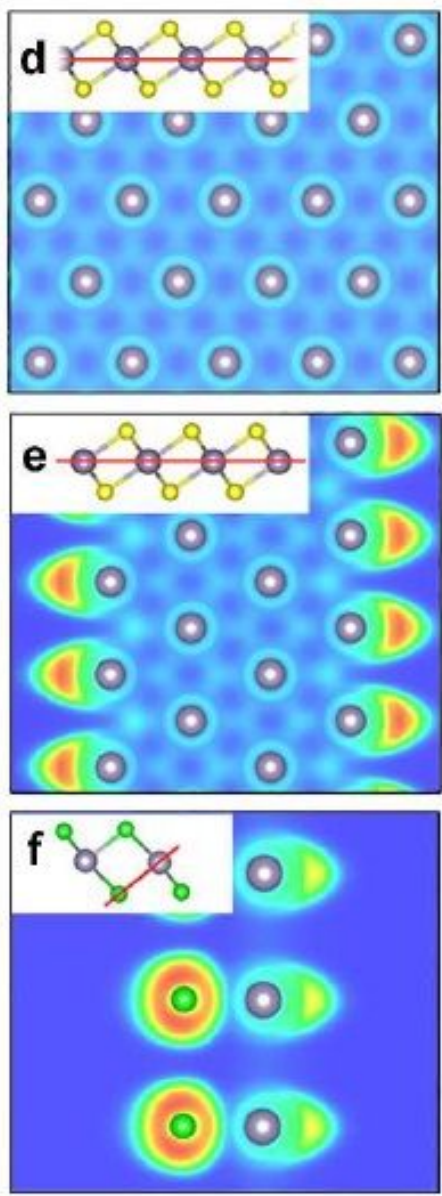

\section{Figure 3}

Self-passivation via LEP on Sn2+. a, Structure of SnS2 monolayer. The blue and red frames denote the structural inheritance of Sn2S3 and SnBr2 from SnS2, respectively. b, c, Structures of Sn2S3 (b) and $\mathrm{SnBr} 2$ (c) atomic chains and their electronic DOS. Fermi level is set to zero. States in the energy range of $-7 \sim-5 \mathrm{eV}$ correspond to the 5s LEP on Sn2+ ions. d-f, ELFs of SnS2 (d), Sn2S3 (e) and SnBr2 (f) in the planes designated by the red lines in the insets. Local maximum of ELF around Sn atom indicates the LEP. 

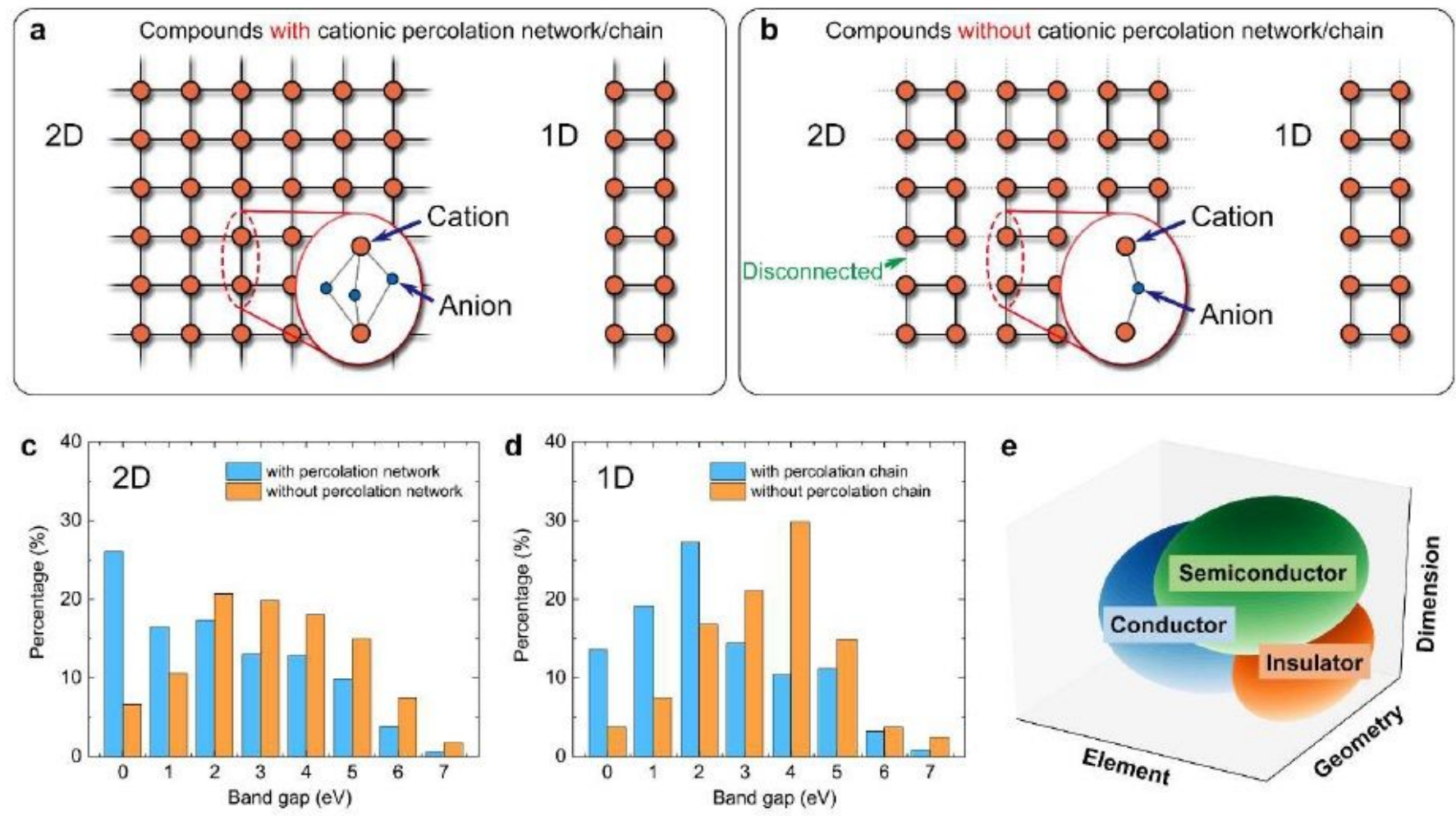

\section{Figure 4}

Influence of cation connectivity on electronic band gap. a, b, Schematic illustration of 2D and 1D structures with (a) or without (b) a cationic percolation network/chain. c, d, The distribution of band gap for 2D (c) and 1D (d) compounds, each divided into two groups by the existence of a cationic percolation network. e, Modulation of electronic structure by tuning constituent element, geometry and dimensionality of the material.

\section{Supplementary Files}

This is a list of supplementary files associated with this preprint. Click to download.

- SI.pdf 